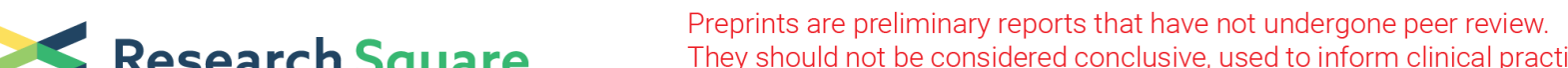 Research Square They should not be considered conclusive, used to inform clinical practice,

\section{Common Breastfeeding Problems and Their Effect on The Continuation of Exclusive or Predominant Breastfeeding in Kinshasa}

Pelagie Babakazo ( $\square$ pelagie.babakazo@unikin.ac.cd)

Universite de Kinshasa Faculte de Medecine https://orcid.org/0000-0001-5561-696X

Marc Bosonkie

Universite de Kinshasa Faculte de Medecine

Eric Mafuta

Universite de Kinshasa Faculte de Medecine

Nono Mvuama

Universite de Kinshasa Faculte de Medecine

Mala-Ali Mapatano

Universite de Kinshasa Faculte de Medecine

Research article

Keywords: Breastfeeding problems, Exclusive breastfeeding, Kinshasa, Democratic Republic of the Congo

Posted Date: July 13th, 2020

DOI: https://doi.org/10.21203/rs.3.rs-36783/v1

License: (9) This work is licensed under a Creative Commons Attribution 4.0 International License.

Read Full License 


\section{Abstract}

Background: Breastfeeding has numerous advantages for infant, mother and society. However, many mothers discontinue breastfeeding due to problems they encounter. This study aimed to identify problems commonly experienced by breastfeeding mothers during the first six months in Kinshasa and to determine their effect on the continuation of exclusive or predominant breastfeeding.

Methods: A prospective cohort study was carried out in Kinshasa from October 2012 to July 2013. A total of 422 mother-infant couples were recruited shortly after being discharged from twelve maternity facilities in Kinshasa and followed-up for six months. Interviews were conducted at the mother's house during the first week after birth, and thereafter at monthly intervals for six months. Data included mother's sociodemographic characteristics, the breastfeeding problems she experienced and information on child's feeding. The Cox Proportional Model was used to determine the effect of breastfeeding problems on the continuation of exclusive or predominant breastfeeding.

Results: Cracked or sore nipples, insufficient production of milk and breast engorgement were the most commonly experienced problems by lactating mothers. The problems occurred mainly during the first week (17.1\%; $\left.\mathrm{Cl}_{95 \%} 13.7-21.1\right)$ and the rest of the first month (16.2\%; $\left.\mathrm{Cl}_{95 \%} 12.8-20.3\right)$. Experiencing breastfeeding problems during any period negatively affected the continuation of exclusive or predominant breastfeeding.

Conclusions: The first month after birth presents the most risk for the occurrence of breastfeeding problems. Those problems negatively affect the course of breastfeeding. Mothers should be supported as soon as possible after delivery, to improve their breastfeeding performance and to be informed on how to maintain breast milk supply.

\section{Background}

Breastfeeding is the optimal way of feeding for neonates and infants. Furthermore, its duration is of vital importance for the child's development and health. The World Health Organization (WHO) recommends that infants be breastfed exclusively for the first six months of life and then, when other foods have been introduced, continue to be breastfed until aged two years or beyond (1). "Exclusive breastfeeding" is defined as feeding the baby with only breast milk, without any other food or drink, not even water (2). "Predominant breastfeeding" on the other hand means that the infant's main source of nourishment is breast milk, while child's diet contains other liquids such as water, water-based drinks or fruit juice (2).

Breastfeeding provides short-term and long-term health and economic advantages to children, women, and society. Those benefits go far beyond nutrition. Children who are breastfed are less at risk of morbidity and mortality, compared to those who are not breastfed (3). Moreover, they are protected against infections and malocclusion, they enjoy a high intelligence quotient, and they may have reduced long-term risk of becoming obese and diabetic (4). For lactating women, breastfeeding ensures spacing of births and prevents breast and ovarian cancers (4). Scaling up breastfeeding to a near universal level 
could prevent 823000 child deaths and 20000 maternal deaths per annum (5). Thus, it contributes to achieving the first and second targets of the third Sustainable Development Goal, related respectively to maternal and child mortalities. Furthermore, not breastfeeding is associated with an economic loss of about $\$ 302$ billion annually or $0.49 \%$ of world gross national income (5).

Despite these advantages, rates of exclusive breastfeeding in most countries remain below the global recommendation. During 2012, the World Health Assembly set a target for 2025 to increase exclusive breastfeeding rates in the first six months up to at least $50 \%$ (6). However, in low-income and middleincome countries, only $37 \%$ of children younger than six months of age are exclusively breastfed (4).

In the Democratic Republic of the Congo (DRC), almost all infants are breastfed and just over half of children less than six months old are exclusively breastfed (7). Although the country is moving towards the 2025 WHO target, from $37 \%$ in 2010 (8) to 52\% in 2018 (7) and exceeding 48\% in 2014 (9), one could hypothesize that the trend would be faster if some obstacles were overcome. According to the WHO, countries that are already at or near $50 \%$ exclusive breastfeeding have to continue to strive for improvements because of the health and economic benefits of exclusive breastfeeding (6). Moreover, despite the improvement noticed during the last decade, the mortality rate among children under five in the DRC remains far from 25\%, the second target of the third Sustainable Development Goal (10). This rate moved from 158\%o in 2010 (8) to 104\%o in 2014 (6) and 70\%o in 2018 (7).

Exclusive breastfeeding is often hindered by a number of problems. A systematic review on obstacles to exclusive breastfeeding in low- and middle-income countries show that breastfeeding problems are commonly reported (11). While most women initiate breastfeeding, many discontinue due to problems encountered rather than maternal choice. Breastfeeding mothers need to be informed about strategies to prevent problems; at the same time, they need support in order to overcome problems they may face. Health care providers have been identified by mothers as the primary source of information on breastfeeding $(12,13)$. Thus, the former could support mothers in preventing breastfeeding problems or managing them if they occurred. This is all the more convincing in a country like the DRC where rates of breastfeeding initiation as well as antenatal visits and delivery in a health care facility are high (7).

Therefore, health care providers should be aware of; common problems experienced by breastfeeding mothers, when they are most likely to occur and whether their effects on the continuation of exclusive or predominant breastfeeding might depend on the moment they occur. This study aimed to identify breastfeeding problems commonly experienced by breastfeeding mothers during the first six months in Kinshasa, to determine when the problems are more frequent as well as their effect on the continuation of exclusive or predominant breastfeeding beyond the moment they occurred.

\section{Methods}

\subsection{Design, setting and participants}


We re-analysed data from a prospective cohort study, on infant feeding practices, carried out among mother-infant couples in Kinshasa from October 2012 to July 2013 . The study design and enrolment of participants are described elsewhere (14). Briefly, mother-infant couples were randomly selected from 12 maternity facilities in Kinshasa during the first week after childbirth. To be eligible, the mother needed to be at least 18 years old, given birth to a single living full-term child who was free of any serious health conditions that would require transfer to an intensive care unit. Each mother-infant couple was followedup during home visits until the infant was fed with non-human milk or with complementary food, or until six months for nursing mothers who had practiced exclusive or predominant breastfeeding for the duration of that period.

\subsection{Definition of concepts}

The researchers in this study used the WHO definitions (2) of breastfeeding and infant feeding practices, as follows: a child was considered exclusively breast-fed, when he or she had received only breast milk with no other liquids (including water) or solids; predominately breast-fed when he or she had received breast milk as the main source of nourishment but also liquids such as water, water-based drinks or fruit juice; and having been complementary fed when that feeding consisted of any food or liquid, including non-human milk and formula, along with breast milk.

The duration of exclusive or predominant breastfeeding was defined as the infant's age at the introduction of non-human milk or complementary foods; in other words, the age at which the infant began complementary feeding. This duration was initially measured in days, and then converted to weeks during data analysis.

\subsection{Data collection}

Face-to-face individual interviews were conducted at the mother's home. Seven interviews were conducted, during the first week after birth and subsequently at monthly intervals for six months. During the first visit, sociodemographic data were collected as follows - mother's age, education level, marital status, main occupation and parity. At each visit, the mother was asked whether she had experienced any

problem or difficulty with breastfeeding since her previous visit or since the delivery for her first visit. If her answer was in the affirmative, she was asked to describe the breastfeeding problems that she had experienced. More than one breastfeeding problem could be reported. The mother was also asked how her infant was fed the previous day. If non-human milk or complementary food was mentioned, the mother was asked when she had started feeding her infant with the food or milk in question.

\subsection{Data analysis}

Characteristics of study participants were summarized using the median and the interquartile range (IQR) for continuous variables and proportions for categorical variables namely: occupation, level of education, marital status and parity. Frequency and type of breastfeeding problems experienced over the six first months were presented in frequency distribution tables. The effect of breastfeeding problems 
encountered during a given period on the continuation of exclusive or predominant breastfeeding beyond this period was determined using the Cox Proportional Model. Crude hazard ratios with their confidence intervals were calculated in bivariate analysis. For these survival analyses, the duration of exclusive or predominant breastfeeding was right censored at the child's age the moment the mother-infant couple dropped out of the study and at six months for mothers who continued exclusive or predominant breastfeeding up to the end of the study. The child's death was also considered as a censoring event. All statistical analysis was performed in Stata version 14. Statistical significance was set at $p<0.05$.

\subsection{Ethical considerations}

The study was approved by the Ethical Committee of the Kinshasa School of Public Health (ESP/CE/001/2012). During the first visit, all participants were informed about the study and the freedom to leave any time should they feel uncomfortable. All research procedures were in accordance with the Declaration of Helsinki.

\section{Results}

\subsection{Participants' sociodemographic characteristics}

A total of 422 mother-infant couples were enrolled in the study. Their sociodemographic characteristics are shown in Table 1. Almost three mothers out of five (57.2\%) were 20 to 29 years old. Their median age was 26 years (IQR 22 to 31). Less than two percent of mothers (1.9\%) had never attended school and nearly half $(49.1 \%)$ had completed primary education. Most of them (85.3\%) were married or living with a partner. Regarding their main occupation, nearly half of the participants $(48.3 \%)$ were housewives. Almost four out of ten participants (38.4\%) were first time mothers. 
Table 1

Characteristics of study participants

\begin{tabular}{|lll|}
\hline & Number & Percent \\
& $\mathbf{n}=\mathbf{4 2 2}$ & \\
\hline Age (years) & & \\
\hline$<20$ & 47 & 11.2 \\
\hline $20-29$ & 241 & 57.2 \\
\hline$\geq 30$ & 133 & 31.6 \\
\hline Education level & & \\
\hline Never been at school & 8 & 1.9 \\
\hline Primary & 207 & 49.1 \\
\hline Secondary & 179 & 42.4 \\
\hline University & 28 & 6.6 \\
\hline Marital status & & \\
\hline Living with a partner & 360 & 85.3 \\
\hline Single & 62 & 14.7 \\
\hline Occupation & & \\
\hline Housewife & 163 & 39.6 \\
\hline Small trade & 204 & 48.3 \\
\hline Hairdresser/dressmaker & 68 & 16.1 \\
\hline Paid job & 20 & 4.7 \\
\hline Student & 106 & 25.1 \\
\hline Farm worker & 6.3 \\
\hline Parity & & \\
\hline 1 & & \\
\hline $2-3$ & & \\
\hline$\geq 4$ & & \\
\hline
\end{tabular}

\subsection{Frequency of breastfeeding problems}

The frequency of breastfeeding problems during different periods, from the first week after delivery to the sixth month is presented in Table 2. The problems were more common during the first month after 
delivery. Their frequency during the first week $(17.1 \% ; 95 \% \mathrm{Cl} 13.7-21.1)$ was similar to that of the rest of the first month $(16.2 \% ; 95 \% \mathrm{Cl} 12.8-20.3)$, and was three times higher than that of the following five months.

Table 2

Proportion of lactating mothers who encountered problems during different periods from delivery to six months

\begin{tabular}{|lll|}
\hline Period & Frequency & Percentage $\left[\mathrm{Cl}_{\mathbf{9 5 \%}}\right]$ \\
\hline $0.0-7.0$ days & $72 / 422$ & $17.1[13.7-21.1]$ \\
\hline $8.0-29$ days & $63 / 389$ & $16.2[12.8-20.3]$ \\
\hline $1.0-1.9$ months & $26 / 388$ & $6.7[4.5-9.8]$ \\
\hline $2.0-2.9$ months & $24 / 366$ & $6.6[4.3-9.7]$ \\
\hline $3,0-3,9$ months & $22 / 326$ & $6.7[4.4-10.2]$ \\
\hline $4,0-4,9$ months & $12 / 206$ & $5.8[3.0-10.0]$ \\
\hline $5,0-5,9$ months & $5 / 107$ & $4.7[1.5-10.6]$ \\
\hline
\end{tabular}

\subsection{Types of breastfeeding problems}

The types of breastfeeding problems are presented in Table 3. Cracked nipples were the most commonly reported breastfeeding problem. Almost one third of mothers reported this problem during the first week $(31.9 \%)$ as well as during the rest of the first month (36.5\%) and beyond the first month $(30.3 \%)$.

Table 3

Type of breastfeeding difficulties encountered by mothers during different periods from delivery to six months

\begin{tabular}{|c|c|c|c|}
\hline Type of breastfeeding problem & 0-7day & $8-29$ day & $\begin{array}{l}s 1,0-5,9 \text { months } \\
n=89\end{array}$ \\
\hline Cracked or wounded nipples & $23(31$. & $23(36.5)$ & $27(30.3)$ \\
\hline Sore nipples during breastfeeding & $22(30.6$ & $12(19.0)$ & $9(10.1)$ \\
\hline Insufficient production of milk & $14(19$. & $10(15.9)$ & $10(11.2)$ \\
\hline Breast engorgement & $5(6.9)$ & $11(17.5)$ & $12(13.5)$ \\
\hline Pain in the operating wound & $4(5.6)$ & 2(3.2) & $1(1.1)$ \\
\hline Difficulty to take the right position & $1(1.4)$ & $3(4.8)$ & $1(1.1)$ \\
\hline Child's sickness & $0(0.0)$ & $3(4.8)$ & $21(23.6)$ \\
\hline Mother's sickness & $0(0.0)$ & 1(1.6) & $4(4.5)$ \\
\hline Breast abscess & $0(0.0)$ & $0(0.0)$ & $4(4.5)$ \\
\hline
\end{tabular}

Apart from cracked nipples, the other most common breastfeeding problems reported during the first month after delivery included sore nipples during breastfeeding, insufficient production of milk and breast engorgement: during the first week, the proportion of mothers who mentioned having breastfeeding problems was respectively $30.6 \%, 19.4 \%$ and $6.9 \%$; whereas during the rest of the month, these proportions declined respectively to $19.0 \%, 15.9 \%$, except for breast engorgement that increased to $17.5 \%$. Beyond the first month, except for the cracked nipples already mentioned (30.2\%), the most breastfeeding problems frequently reported included infant's illness (23.6\%), insufficient production of milk (11.2\%) and 
sore nipples during breastfeeding (10.1\%).

\subsection{Effects of breastfeeding problems}

With regard to the consequences of breastfeeding problems on the continuation of exclusive or predominant breastfeeding, this study showed that mothers who had experienced breastfeeding problems during a given period were more likely to discontinue exclusive or predominant breastfeeding beyond this period (Table 4). This association, found throughout the first six months except during the third ( $\left.\mathrm{HR}=0.1 ; \mathrm{Cl}_{95 \%} 0.0-23.7\right)$ and fourth months $\left(\mathrm{HR}=0.8 ; \mathrm{Cl}_{95 \%} 0.3-1.9\right)$, was higher during the first month $\left(\mathrm{HR}=6.7 ; \mathrm{Cl}_{95 \%} 1.8-24.9\right)$, followed by the sixth $\left(\mathrm{HR}=5.7 ; \mathrm{Cl}_{95 \%} 2.1-15.1\right)$ and the second $(\mathrm{HR}=$ 3.7; $\mathrm{Cl}_{95 \%}$ 1.0-13.1) months.

\section{Table 4}

Effect of problems encountered during a given period on the noncontinuation of exclusive or predominant breastfeeding

\begin{tabular}{|c|c|c|c|c|c|c|}
\hline \multirow{3}{*}{\begin{tabular}{|l} 
Period \\
(months \\
$0.0-0.9$
\end{tabular}} & \multicolumn{5}{|c|}{ Breastfeeding problemsEventsPersons-weeks HR* $\left(\mathrm{Cl}_{95 \%}\right)$} & \multirow{2}{*}{\begin{tabular}{|l} 
p-value \\
0.005
\end{tabular}} \\
\hline & yes & 63 & 5 & 243 & $6.7(1.8-24.9$ & \\
\hline & No & 326 & 4 & 1295 & 1 & \\
\hline $1.0-1.9$ & yes & 26 & 3 & 202 & $3.7(1.0-13.1)$ & 0.043 \\
\hline & No & 362 & 12 & 2883 & 12 & \\
\hline $2.0-2.9$ & yes & 24 & 0 & 288 & $0,1(0,0-23.7)$ & 0.332 \\
\hline & No & 342 & 30 & 4087 & 1 & \\
\hline $3.0-3.9$ & yes & 22 & 5 & 345 & $0,8(0,3-1.9)$ & 0.554 \\
\hline & No & 304 & 90 & 4787 & 1 & \\
\hline $4.0-4.9$ & yes & 12 & 8 & 227 & $2.2(1.1-4.5)$ & 0.036 \\
\hline & No & 194 & 79 & 4785 & & \\
\hline $5.0-5.9$ & yes & 5 & 5 & 109 & $5.7(2.1-15.1$ & 0.001 \\
\hline & No & 102 & 63 & 7696 & 1 & \\
\hline
\end{tabular}

\section{Discussion}

This study showed that cracked nipples, sore nipples during breastfeeding, insufficient production of milk and breast engorgement were the breastfeeding problems most commonly encountered by lactating mothers. These breastfeeding problems occurred mainly during the first week after birth and throughout the rest of the first month. Breastfeeding problems during a given period negatively impacted on exclusive or predominant breastfeeding continuing beyond that time.

During this study, the highest frequency of breastfeeding problems was noted within the first week after delivery $(17.1 \%)$ and remained high during the rest of the first month (16.2\%) but decreased from the second month (6.7\%). At six months, less than one out of twenty breastfeeding mothers $(4.7 \%)$ reported experiencing breastfeeding problems. These results reinforce the evidence that the first month after childbirth, and especially the first week, is critical for the establishment and the continuation of breastfeeding (15). This observation suggests that the mother should be supported during this period to prevent and overcome breastfeeding difficulties. Higher proportions of breastfeeding problems (40-80\%) have been reported during the first week in developed countries (16-18). An explanation for this noticeable difference is the fact that breastfeeding is common in Africa; therefore, African women could be positive thinkers towards breastfeeding. Women who think positively about breastfeeding perceive 
problems as 'normal' while those who lack self-assurance in their capacity to breastfeed are more likely to focus on its negative aspects (19).

With regard to the type of breastfeeding problems encountered, the most reported were: cracked nipples; sore nipples during breastfeeding; insufficient production of breast milk; and breast engorgement. The same problems were also reported during the first month, with almost similar proportions in Denmark (17), the United States of America (USA) (18) and Ethiopia (20). Cracked or sore nipples are mainly a consequence of poor breastfeeding technique (21) that can be prevented with good positioning, optimal attachment of the baby and gentle removal from the breast when the baby is satisfied $(16,22)$. Therefore, breastfeeding technique should be assessed and taught to each mother at least once by midwives while at the maternity facility and corrected if needed (22).

Apart from cracked or sore nipples, insufficient production of breast milk was the second most common breastfeeding problem reported by mothers during this study. However, this insufficiency is more often secondary than primary (16). The primary glandular insufficiency is rare. More often, insufficient milk production occurs: when the breasts are not emptied sufficiently and frequently for whatever reason, notably poor breastfeeding technique; painful latch on by suckling; mother and infant separation; and illness of mother or infant (22). If the mother and infant are separated or unwell, early and regular milk expression by hand or pump should be started to maintain milk production (23). On the other hand, insufficient production of breast milk is more perceived than real (19). The mother's perception of not having enough milk is often a misinterpretation of her crying baby or a feeling of soft or empty breasts. Therefore, the mother experiencing insufficient production of breast milk needs to be supported by midwives and relatives to improve her breastfeeding self-efficacy.

The third most common breastfeeding problem reported in this study was breast engorgement that, once again, resulted from a poor emptying of breasts (16). Early initiation of breastfeeding, spending more time to breastfeed during the first 48 hours after childbirth and emptying one breast at each breastfeeding by alternating the breast that is first offered, may help to prevent this problem $(22,24)$.

This study pointed out that breastfeeding problems during a given period had a negative impact on the continuation of exclusive or predominant breastfeeding beyond this period, except during the third and fourth months. The fact that no association was found during the third and fourth months of life suggests that weaning was probably planned at this age. Indeed, mixed feeding was the norm among older generations which prefer that the current mothers adopt the same infant feeding practices (25). Before 2001, the WHO allowed the introduction of complementary foods from the third to fourth months (26).

Regarding the strength of the association, the highest hazard ratio was noted during the first month after delivery. During this period, the hazard of discontinuing exclusive or predominant breastfeeding was seven times higher among mothers who had experienced breastfeeding problems. This result highlights, once again, that the first weeks after childbirth are critical to the establishment and the continuation of breastfeeding. Similar findings were reported in Sweden (27) and USA (18). 
In addition, this study noted also a high hazard ratio during the sixth month of life; mothers who experienced breastfeeding problems were six times more at risk to stop exclusive or predominant breastfeeding. This may suggest that these mothers had planned probably to breastfeed exclusively for the first six months but could not reach their goal due to breastfeeding problems. These results illustrate the importance of breastfeeding counselling, which should begin during the antenatal period and continue throughout the breastfeeding period, particularly the first six months.

\subsection{Strengths and limitations}

The results of this study should be interpreted taking into account its strengths and limitations.

One of the limitations was that the data used were collected in 2013 and may seem outdated. However, in our opinion, results from this study are still valuable today, given that the rate of mothers achieving the global recommendation on exclusive breastfeeding remained around 50\% from 2013 to 2018 . This study was prospective; thus, the low reliability of the duration of exclusive breastfeeding determined retrospectively was avoided. However, the recall bias was not completely avoided because the day when foods or drinks other than breast milk were introduced was determined retrospectively. Therefore, mothers could or could not remember precisely that moment. Nonetheless, to our knowledge, this study is the first carried out in Kinshasa to investigate breastfeeding problems experienced by mothers.

\subsection{Recommendation for future research}

The most common breastfeeding problems reported during this study may be prevented by improving the breastfeeding technique. Furthermore, these problems occurred mainly during the first month after the childbirth, especially during the first week. Future research should be carried out to assess the effect of technical support, offered as early as possible after the childbirth to improve the breastfeeding technique, on the occurrence of these problems.

\section{Conclusion}

This study has demonstrated that lactating mothers experience several problems. The first four weeks after birth are the most critical to the occurrence of those problems. When problems occur, they negatively affect the continuation of exclusive or predominant breastfeeding. Most of these problems might be prevented with optimal breastfeeding technique and by emptying sufficiently and frequently the breasts. Therefore, breastfeeding mothers should be supported as soon as possible after delivery, to improve their breastfeeding technique and to be informed on how to maintain breast milk supply.

\section{List Of Abbreviations}

Cl: Confidence interval, DRC: Democratic Republic of the Congo, HR: Hazard ratio, IQR: Interquartile range, WHO: World Health Organization.

\section{Declarations}




\section{Ethics approval and consent to participate}

The study was approved by the Ethical Committee of the Kinshasa School of Public Health, University of Kinshasa (Ethics Certificate Clearance Number: ESP/001/2012). Mothers who were interviewed in this study were at least 18 years old, they gave their written informed consent to participate.

\section{Consent to publish}

The consent was sought from study participants. The consent forms stated that the aggregated results would be shared locally, at academic conferences, and in journal articles.

\section{Availability of data and materials}

The datasets used and/or analysed during the current study are available from the corresponding author on reasonable request.

\section{Competing Interests}

All authors declare that they have no commercial or other associations that may pose a conflict of interest.

\section{Funding}

Not applicable. No funding was received.

\section{Authors' contributions}

PB designed the study, analysed and interpreted the data, and drafted the manuscript. MB, EM, NM and MM made substantial revision of the manuscript. All authors read and approved the final version.

\section{Acknowledgements}

We would like to thank the mothers who participated in this study and who shared their valuable time with us. We also greatly appreciate the support and full commitment of the interviewers during this study.

\section{References}

1. OMS. Stratégie mondiale pour l'alimentation du nourrisson et du jeune enfant. Genève: OMS; 2003.

2. WHO. Indicators for assessing infant and young child feeding practices. Geneva: WHO; 2008.

3. Horta BL, Bernardo L, Victora G, Cesar G, WHO. Short-term effects of breastfeeding: a systematic review on the benefits of breastfeeding on diarrhoea and pneumonia mortality. Geneva: WHO; 2013.

4. Victora CG, Bahl R, Barros A, França G, Horton S. Breastfeeding in the 21st century: epidemiology, mechanisms, and lifelong effect. Lancet. 2016;387:475-90. 
5. Rollins NC, Bhandari N, Hajeebhoy N, Horton S, Lutter CK, Martines JC, et al. Why invest, and what it will take to improve breastfeeding practices? Lancet. 2016;387:491-504.

6. WHO/UNICEF. Global nutrition targets 2025: breastfeeding policy brief. Geneva: World Health Organization; 2014.

7. INS/RDC. Enquête par grappes à indicateurs multiples, 2017-2018. Kinshasa: INS/RDC; 2019.

8. MiniPlan/RDC. Enquête par grappe à indicateurs multiples 2010 (MICS-2010). Kinshasa: MiniPlan; 2010.

9. MiniPlan/MiniSanté. Enquête Démographique et de Santé en République Démocratique du Congo 2013-2014. Kinshasa; 2014.

10. UNDP. Sustainable Development Goals. New-York: UNDP; 2015.

11. Kavle J, LaCroix E, Dau H, Engmann C. Addressing barriers to exclusive breast-feeding in low- and middle-income countries: a systematic review and programmatic implications. Public Health Nutr. 2017;20(17):3120-34.

12. Mushaphi L, Mahopo T, Nesamvuni C, Baloyi B, Mashau E, Richardson J, et al. Recommendations for infant feeding policy and programs in Dzimauli Region, South Africa: Results from the MAL-ED Birth Cohort. Food Nutr Bull. 2017;38(3):428-40.

13. Asare B, Preko J, Baafi D, Dwumfour-Asare B. Breastfeeding practices and determinants of exclusive breastfeeding in a cross-sectional study at a child welfare clinic in Tema Manhean, Ghana. Int Breastfeed J. 2018;13:12.

14. Babakazo P, Donnen P, Akilimali P, Ali NM, Okitolonda E. Predictors of discontinuing exclusive breastfeeding before six months among mothers in Kinshasa: a prospective study. Int Breastfeed J. 2015;10:19.

15. Semenic S, Loiselle C, Gottlieb L. Predictors of the duration of exclusive breastfeeding among firsttime mothers. Res Nurs Health. 2008;31(5):428-41.

16. Bergmann RL, Bergmann KE, von Weizsacker K, Berns M, Henrich W, Dudenhausen JW. Breastfeeding is natural but not always easy: intervention for common medical problems of breastfeeding mothers - a review of the scientific evidence. J Perinat Med. 2014;42(1):9-18.

17. Feenstra MM, Jorgine Kirkeby $M$, Thygesen $M$, Danbjorg DB, Kronborg H. Early breastfeeding problems: A mixed method study of mothers' experiences. Sex Reprod Healthc. 2018;16:167-74.

18. Wagner EA, Chantry CJ, Dewey KG, Nommsen-Rivers LA. Breastfeeding concerns at 3 and 7 days postpartum and feeding status at 2 months. Pediatrics. 2013;132(4):e865-75.

19. Meedya S, Fahy K, Kable A. Factors that positively influence breastfeeding duration to 6 months: a literature review. Women Birth. 2010;23:135-45.

20. Nkala TE, Msuya SE. Prevalence and predictors of exclusive breastfeeding among women in Kigoma region, Western Tanzania: a community based cross-sectional study. Int Breastfeed J. 2011;6(1):17.

21. Niazi A, Rahimi V, Soheili-Far S, Askari N, Rahmanian-Devin P, Sanei-Far Z, et al. A systematic review on prevention and treatment of nipple pain and fissure: Are they curable? J Pharmacopuncture. 
2018;21(3):139-50.

22. WHO, UNICEF. Breastfeeding promotion and support in a Baby-Friendly Hospital-20 hour Course. Geneva: WHO; 2006.

23. Amir LH. Managing common breastfeeding problems in the community. BMJ. 2014;348:g2954.

24. Moon J, Humenick S. Breast engorgement: contributing variables and variables amenable to nursing intervention. J Obstst Gynecol Neonat Nurs. 1989;18:309-15.

25. Otoo GE, Lartey AA, Perez-Escamilla R. Perceived incentives and barriers to exclusive breastfeeding among periurban Ghanaian women. J Hum Lact. 2009;25(1):34-41.

26. WHO. World Health Organisation's infant feeding recommendation. Weekly Epidemiological Record. $1995 ; 70$.

27. Gerd AT, Bergman S, Dahlgren J, Roswall J, Alm B. Factors associated with discontinuation of breastfeeding before 1 month of age. Acta Paediatr. 2012;101(1):55-60.

\section{Supplementary Files}

This is a list of supplementary files associated with this preprint. Click to download.

- BabakazoBreastfeedingProblemsDatacollectiontool.docx 